\title{
Ceftriaxone-induced PSEUdolithiasis: NOt JUSt A theORETICAL RISK
}

Singapore Med J 2017; 58(11): 676-677 doi: 10.11622/smedj.2017103

Dear Sir,

Ceftriaxone is a commonly prescribed third-generation cephalosporin. The potential risk of biliary pseudolithiasis following the use of ceftriaxone, although very rare, is certainly not limited to the neonatal age group. The clinical course of this seemingly infrequent side effect needs to be recognised in order to avoid multiple blood tests and radiological investigations, which may cause anxiety for patients and parents. We report a unique case of ceftriaxone-induced pseudolithiasis in a previously well five-month-old girl, who received two doses of intravenous (IV) ceftriaxone.

Our patient was diagnosed with left-sided vesicoureteric junction obstruction and Escherichia coli-associated pyelonephritis. She received two doses of IV ceftriaxone $(50 \mathrm{mg} / \mathrm{kg} /$ dose daily). The was changed to IV co-amoxiclav when the urine culture result was known. Her fever had settled by then and she remained well. On the third day after stopping ceftriaxone, she developed pale stools, which persisted for four days. She had no history of neonatal jaundice and was not on any other medication. On physical examination, she was found to be thriving with no clinical jaundice or hepatosplenomegaly. Liver function tests showed transaminitis (aspartate aminotransferase [AST] 140 [normal range 20-65] U/L and alanine aminotransferase [ALT] 321 [normal range 10-40] U/L) and elevated gamma-glutamyl transferase (GGT) of 814 (normal range 15-110) U/L. Ultrasonography of the hepatobiliary system showed multiple mobile echogenic foci within the gallbladder with moderately thickened gallbladder wall, which suggested biliary sludge/gallstones (Fig. 1a). The biliary tree was not dilated, with the common bile duct measuring 15 mm in diameter. No choledochal cyst or biliary tree abnormality was detected. The liver appeared normal in outline and echogenicity. Her stool colour, which was closely monitored, became pigmented again on the sixth day after stopping IV ceftriaxone. Liver function test performed 20 days later

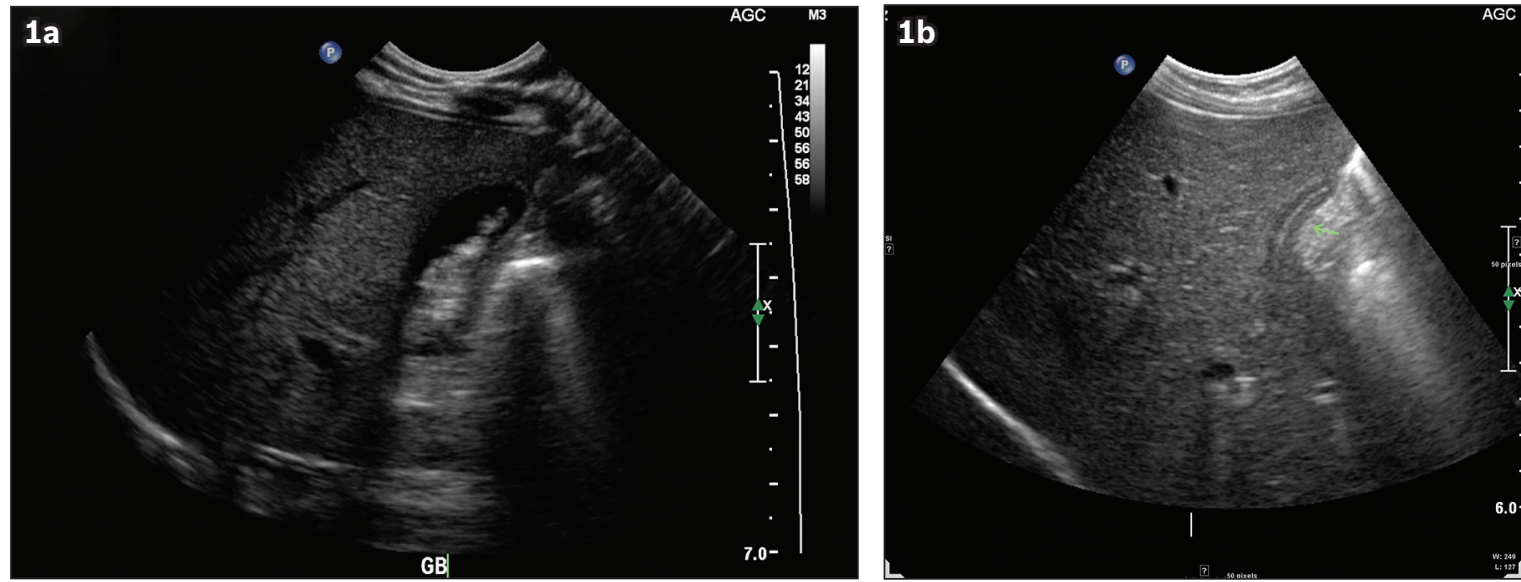

Fig. 1 US images of the hepatobiliary system (a) at presentation shows the gallbladder with multiple mobile echogenic foci suggestive of biliary sludge/gallstones and (b) nine days later shows a collapsed gallbladder with no definite calculi seen within.

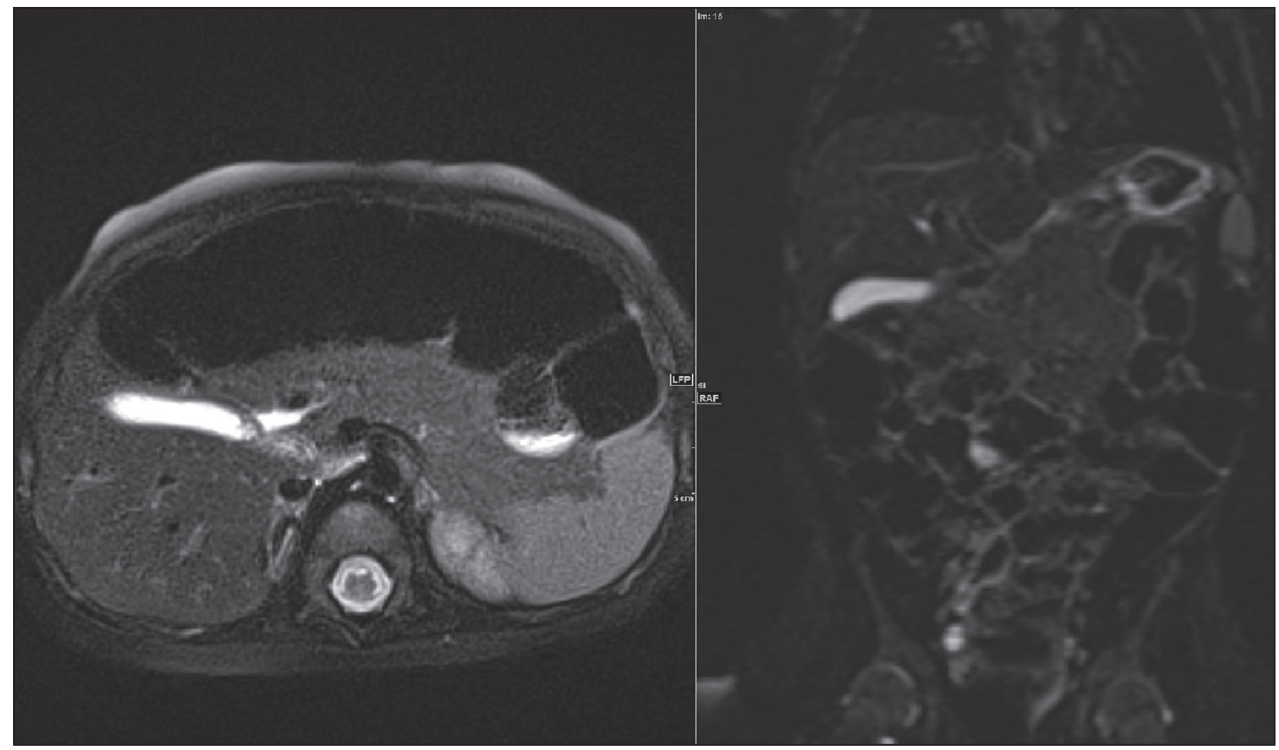

Fig. 2 MR images show a normal gallbladder with no cholelithiasis. 
showed normalisation of liver enzymes - AST $44 \mathrm{U} / \mathrm{L}$, ALT $23 \mathrm{U} / \mathrm{L}$ and GGT $75 \mathrm{U} / \mathrm{L}$. Repeat ultrasonography of the hepatobiliary system performed nine days later showed an empty and contracted gallbladder (Fig. 1b). Magnetic resonance imaging done at the same time to investigate her underlying urinary tract abnormality also showed normal gallbladder with no cholelithiasis (Fig. 2).

Ceftriaxone-associated pseudolithiasis was first described in 1988 by Schaad et al. ${ }^{(1)}$ Once ceftriaxone is administered, approximately $40 \%$ of the administered dose is excreted unmetabolised in the bile, while the rest is excreted in the urine. ${ }^{(2)}$ The potential risk of ceftriaxone causing biliary pseudolithiasis is probably due to its precipitation in the gallbladder. ${ }^{(3)}$ The incidence of ceftriaxone-induced pseudolithiasis was reported to be $25 \%(\mathrm{n}=11 / 44)$ in a study by Papadopoulou et al. ${ }^{(4)}$ In $6(54 \%)$ of these 11 children, pseudolithiasis developed within three days of ceftriaxone administration. ${ }^{(4)}$ Previous studies have not shown any strong evidence with regard to whether ceftriaxone administration in divided doses is associated with reduced biliary sludge formation. A similar result was seen when bolus dosing was compared to slow IV infusion. ${ }^{(3)}$ Previous studies have also shown that pseudolithiasis is more frequent in older children, when ceftriaxone is given at higher doses and for a longer duration of treatment. ${ }^{(3,4)}$ The majority of these children were asymptomatic, although some presented with right-sided abdominal pain and/or abdominal distension. ${ }^{(1,4)}$

To the best of our knowledge, our patient is the first to present with acholic stools. A recent study suggested that a short period of fasting and bed rest are both associated with biliary pseudolithiasis. ${ }^{(5)}$ Ensuring adequate hydration may help to reduce the occurrence of this side effect. The mean duration to resolution of biliary pseudolithiasis is about 15 days. ${ }^{(6)}$ Cessation of ceftriaxone was the only treatment necessary in all reported cases. ${ }^{(1,3,4)}$ However, one patient was reported to require laparoscopic cholecystectomy subsequent to biliary pseudolithiasis secondary to ceftriaxone treatment. ${ }^{(7)}$ Physicians and radiologists need to be aware of this potential adverse effect so as to avoid unnecessary investigations.

Yours sincerely,

Lijia $\underline{\text { Fan }}^{1}$, Perry Lau ${ }^{1}$, Jeevesh $\underline{K a p u r}^{2}$, Sivaramakrishnan Venkatesh Karthik ${ }^{1}$

${ }^{1}$ Department of Paediatrics, ${ }^{2}$ Department of Diagnostic Imaging, National University Health System, Singapore. Li_jia_fan@nuhs.edu.sg

\section{REFERENCES}

1. Schaad UB, Wedgwood-Krucko J, Tschaeppeler H. Reversible ceftriaxone-associated biliary pseudolithiasis in children. Lancet 1988; 2:1411-3.

2. Arvidsson A, Alván G, Angelin B, Borgå O, Nord CE. Ceftriaxone: renal and biliary excretion and effect on the colon microflora. J Antimicrob Chemother 1982; 10:207-15.

3. Soysal A, Eraşov K, Akpinar I, Bakir M. Biliary precipitation during ceftriaxone therapy: frequency and risk factors. Turk J Pediatr 2007; 49:404-7.

4. Papadopoulou F, Efremidis S, Karyda S, et al. Incidence of ceftriaxone-associated gallbladder pseudolithiasis. Acta Paediatr 1999; 88:1352-5.

5. Murata S, Aomatsu T, Yoden A, Tamai H. Fasting and bed rest, even for a relatively short period, are risk factors for ceftriaxone-associated pseudolitiasis. Pediatr Int 2015; 57:942-6.

6. Kim YS, Kestell MF, Lee SP. Gall-bladder sludge: lessons from ceftriaxone. J Gastroenterol Hepatol 1992; 7:618-21.

7. Alemayehu H, Desai AA, Thomas P, Sharp SW, St Peter SD. Ceftriaxone-induced pseudolithiasis in children treated for perforated appendicitis. Pediatr Surg Int 2014; 30:323-6. 